\title{
ACTUALIZACIÓN HIPOTIROIDISMO EQUINO
}

\section{JOSÉ HENRY OSORIOํㅜ, FELIPE RAMÍREZ ECHEVERRY²}

Recibido el 13 de abril de 2012 y aprobado el 13 de marzo de 2013

\section{RESUMEN}

Objetivo: Realizar una actualización en hipotirodismo en equinos. Materiales y Métodos: Se analizó la literatura disponible de los últimos 50 años en las bases de datos BBCSLILACS, Fuente Académica, IB-PsycINFO, IB-SSCI, IB-SciELO, Scopus y Scirus, al igual que artículos históricos, textos y referencias citadas en trabajos públicos. Resultados: Se obtuvo información pertinente relacionada con los objetivos propuestos en la presente revisión, por lo cual puede clasificarse en 7 secciones a saber: etiología, signos clínicos, diagnóstico, factores que alteran las pruebas diagnósticas, síndrome de enfermedad no tiroidea, prevención y tratamiento. Conclusión: El hipotiroidismo una de las alteraciones endocrinas más comunes en los equinos, los signos clínicos son muy característicos incluyendo letargia, retraso en el crecimiento, debilidad e incoordinación. El método diagnóstico más exacto es la medición de las concentraciones de T4L en suero por diálisis. La levotiroxina sódica continúa siendo el tratamiento de elección para el hipotiroidismo en equinos.

\section{PALABRAS CLAVE}

Equinos, hormona tiroidea, metabolismo.

\section{ABREVIATURAS}

TSH, hormona estimulante de la tiroides; HPT, eje hipotálamopituitario; T4, tetrayodotiroxina; T4T, tetrayodotiroxina total; $\mathrm{TRH}$, factor liberador de tiropropina; THs, hormonas tiroideas; T3T, triyodotiroxina; T4L, tetrayodotiroxina libre.

\section{EQUINE HYPOTHYROIDISM: AN UPDATE}

\section{ABSTRACT}

Objective: To carry out an update on equines hypothyroidism. Material and Methods: Available literature from the last 50 years included in the BBCS-LILACS, Fuente Académica, IBPsycINFO, IB-SSCI, IB-SciELO, Scopus and Scirus, data base as well as historical articles, texts and references cited in work published to date were analyzed. Results: Important information related to the objectives proposed in the present review were found and analyzed. It was then divided in seven sections as follows: etiology, clinical signs, diagnosis, factors which alter the diagnostic tests, no thyroid disease syndrome, prevention and treatment. Conclusion: Hypothyroidism is one of the most common alterations in equines; clinical signs are very 
characteristic including lethargy, failure to thrive, debility and lack of coordination problems. The most exact diagnostic method is serum measurement of $\mathrm{T} 4 \mathrm{~L}$ concentration per dialysis. Levothyroxine sodium continues to be the ideal treatment for equine hypothyroidism.

\section{KEY WORDS}

Equines, thyroid hormone, metabolism.

\section{ABREVIATIONS:}

TSH, thyroid stimulating hormone; HPT hypothalamic- pituitary axis; T4 tetra-iodothyronine; T4T Total tetra-iodothyronine; TRH Thyrotropin-releasing hormone; THs Thyroid hormones; T3T type 3 iodothyronine; T4L, tetra-iodothyronine libre.

\section{INTRODUCCIÓN}

Dentro de las patologías que involucran la glándula se encuentran el hipotiroidismo (disminución en la producción de hormonas por parte de la glándula) y el hipertiroidismo (exceso en la producción de hormonas por parte de la glándula). Dichos estados patológicos pueden suceder tanto en animales jóvenes como en adultos; estas alteraciones con respecto a los niveles basales producen un crecimiento muy rápido o muy lento del animal, anomalías en la formación de la estructura ósea que con el paso del tiempo se ven reflejadas tanto en fracturas espontáneas como en la degeneración de estructuras óseas especialmente articulares (rodillas, corvejones) (Hurcombe, 2011). Se define como una deficiencia de hormona del tiroides ( $\mathrm{TH}$, por sus siglas en inglés), de actividad tiroidea o una disrupción en el eje pituitario talámico (HPT) (Breuhaus \& LaFevers, 2005). La expresión de síndrome metabólico equino (SME), fue utilizada por primera vez por Johnson en 2002, para aproximarse de una mejor manera a una condición que anteriormente venía siendo atribuida al hipotiroidismo (Frank, Sojka \& Messer, 2002)Se deducía que los animales sufrían de hipotiroidismo puesto que ganaban peso fácilmente, se volvían obesos, y tenían grandes depósitos de tejido adiposo en el cuello y en la región de la base de la cola. Además de arrojar resultados de T4T por debajo de los índices de normalidad; sin embargo, los signos anteriormente mencionados no fueron confirmados a través de las investigaciones pues los caballos tiroidectomizados no desarrollaron obesidad, depósitos anormales de tejidos adiposos o laminitis (Frank et al., 2005; Breuhaus, 2011)Por lo tanto, las bajas concentraciones de T4 libre son una consecuencia y no una causa de SME en caballos, este hallazgo no es más que un indicador del descontrol metabólico y endocrino que el animal está presentando (Hurcombe, 2011). En algunas ocasiones se encuentran menos concentraciones de T4 en suero en caballos que sufren de SME, este hallazgo se correlaciona con las concentraciones de insulina en el plasma donde se reportó una 
débil correlación negativa ( $r \quad 1 / 4 \quad-0,2 ; \quad P<0,001)$ en las concentraciones de insulina y T4 en caballos con SME (Hurcombe, 2011). El síndrome metabólico varía en los signos clínicos, el principal es la obesidad especialmente visceral, insulinorresistencia, dislipidemia e hipertensión. En caballos y ponis susceptibles a laminitis se ha descrito un genotipo insulinorresistente que semeja al síndrome metabólico en humanos (Malinowski et al., 1996; Geor, 2008). Sin embargo, aunque el síndrome metabólico se relaciona con obesidad y laminitis y al afectarse la función pituitaria se alteran los niveles tiroideos, los síntomas de este síndrome corresponden principalmente a un hiperadrenocortisismo e insulinorresistencia y no a un hipotiroidismo, es por ello que el tratamiento de este síndrome no incluía la administración de suplementos de TH (Johnson, PJ 2002).

\section{ETIOLOGÍA}

El hipotiroidismo puede resultar debido a la remoción del tejido tiroideo o a la supresión de la tiroides. Se clasifica en primario, secundario o terciario (Hurcombe, 2011). Hipotiroidismo primario: es la disfunción propia de la glándula, puede ser causado por una deficiencia de yodo (bocio endémico) o un exceso de yodo (efecto Wolff-Chaikoff), tiroiditis, neoplasia, defectos bioquímicos, agenesia tiroidea o ingestión de componentes goitrogénicos los cuales pueden bloquear la síntesis hormonal (Hurcombe, 2011). Hipotiroidismo secundario (central): resulta de la disfunción hipotalámica o pituitaria. Los autores refieren hipotiroidismo por adenoma pituitario, pero todos los caballos con esta alteración no presentan hipotiroidismo concomitante (Hurcombe, 2011). Hipotiroidismo terciario: denota un defecto en el uso de la hormona por parte de los tejidos periféricos. Esta condición no ha sido reportada en caballos (Johnson, CA 2002). El hipotiroidismo puede deberse a enfermedades adyacentes que afectan la función tiroidea, al igual que componentes exógenos que afectan el tejido tisular e interfieren con la síntesis de TSH. Menos frecuente ocurre por desórdenes que afectan el hipotálamo o la glándula pituitaria (Hyypp, 2005). El hipotiroidismo secundario puede deberse a una supresión de TH u hormona liberadora tiroidea (TRH, por sus siglas en inglés) (Sojka, 1993). Un número de factores no tiroideos afectan potencialmente el eje HPT afectando los niveles de TH circulantes, como la fenilbutazona, dietas con altos contenidos de carbohidratos, proteínas y zinc, administración de glucocorticoides, privación de comida, estado de preñez e ingestión de pasto contaminado con hongos (Frank, 2009). Fármacos como los esteroides sexuales, alteran la función tiroidea; los esteroides gonadales ejercen influencia sobre la acción primaria de la tiroides perturbando la producción de tiroglobulina (TBG, por sus siglas en inglés) (Rudi's, R'onai \& Bartha, 2005). Como el suministro de estrógenos produce un incremento en las concentraciones de TBG en suero, la terapia con andrógenos resulta en una disminución en TBG. Los efectos de los esteroides gonadales sobre la TBG son modulados por la estructura química de los 
mismos según el modo y la vía de administración (Tahboud \& Arafah, 2009). Se han identificado altos niveles de nitrato en la dieta como posible causa de hipotiroidismo congénito, ya que los nitratos pueden alterar la función de la glándula tiroides y pueden atravesar la placenta en diferentes especies; algunos alimentos verdes pueden concentrar altos niveles de nitratos y nitritos como la alfalfa, pasto de centeno o timoteo y cultivos de cereales como trigo, avena y cebada, los cuales a su vez poseen niveles muy bajos de yodo (Johnson CA, 2002). Por otro lado, los nitratos también pueden presentar altas concentraciones de agua, particularmente en áreas donde se usan altas cantidades de fertilizantes, engordas y lecherías (Hyypp, 2005). El hipotiroidismo en potros tiene etiología nutricional y se ha asociado a un largo periodo de gestación (Roser, 2008). El aumento de tamaño de la tiroides con una disminución en la función, es asociado con una inadecuada captación del yodo y una excesiva toma de yodo por parte de la madre. La tiroxina es transportada por vía transplacentaria y por la leche. No existen casos de hipotiroidismo producidos por errores innatos en el metabolismo de los potros (Toribio \& Duckett, 2004). La literatura reporta que el bocio y el hipotiroidismo se asocian a una ingestión excesiva de yodo en las raciones suplementarias, equivalentes a $40 \mathrm{mg}$ o más de yodo al día (Ahmed et al., 2008). Estudios realizados han demostrado que el hipotiroidismo en los potros está influenciado por la dieta de la madre durante la gestación, donde se encontró que una deficiencia en suplementos minerales como el yodo y los nitratos, elementos que interfieren con la función tiroidea, son un factor predisponente para la presentación del síndrome de inmadurez en potros (Hyypp, 2005).

\section{SIGNOS CLÍNICOS}

Los principales signos reportados en la deficiencia de $\mathrm{TH}$ en equinos son letargia, intolerancia al ejercicio y una delgada capa de pelo (Allen et al., 1996). Las hembras con hipotiroidismo presentan múltiples dificultades reproductivas, incluyendo anovulación, infertilidad, abortos y preñeces complicadas (Johnson CA, 2002). En los caballos algunos signos clínicos secundarios a la disminución de la función tiroidea son similares a la anhidrosis. Sin embargo, se ha reportado que caballos tireidectomizados sudan normalmente (Hulbert, 2000). Dentro de la sintomatología los caballos hipotiroideos después de realizar algún esfuerzo físico pueden presentar calambres, espasmos, debilidad muscular, en algunos casos se manifiesta intolerancia al ejercicio acompañada por ataques repetitivos de azoturia, sudoración difusa, mioglobinuria, mialgia, tremor muscular y una actitud general conocida como "cretinismo" en la cual el animal tiene poca disposición para realizar sus ejercicios y una capacidad de aprendizaje menor a la que normalmente presentan los caballos en promedio (Geor, 2010). En los caballos que son tiroidectomizados jóvenes se observan signos como una disminución en la tasa de crecimiento, en el cierre de las placas fiseales y en la erupción dental, insensibilidad fría, capa de pelo 
grueso, piel de la cara engrosada, disminución de la temperatura rectal, disminución de las concentraciones en suero de fósforo e incremento de colesterol. Sin embargo, el semen de los machos es fértil y las yeguas pueden quedar preñadas y llevar los potros a largo plazo (Breuhaus \& LaFevers, 2005). Es de frecuencia la presentación de laminitis debido al síndrome metabólico equino el cual representa alteración en la glándula pituitaria, sin embargo se asocia principalmente con hiperadrenocortisismo lo cual como es sabido disminuye las defensas por el exceso de glucocorticoides (McLaughlin, Doige \& McLaughlin, 1986) agravando fuertemente el cuadro de laminitis en equinos, de igual forma al disminuir el gasto de oxígeno por la disminución en TH favorece la proliferación bacteriana y en general microbiana presente en esta alteración, estos caballos se caracterizan por una apariencia obesa y una mala distribución de las grasas (Johnson et al., 2004). Dentro de la fallas fisiológicas que genera el hipotiroidismo en las hembras lo quistes ováricos son una de sus principales, esto ocurre como consecuencia de un aumento de la captación de yodo por parte de los ovarios, la cual es inducida por el tiouracilo (Johnson et al., 2004). En humanos la formación de ovarios quísticos es muy común en mujeres que padecen de hipotiroidismo primario (Himler et al., 2010). Los caballos comienzan a ganar peso con poca alimentación y tienden a acumular grasa en la cresta del cuello y sobre la grupa (Symonds, 1995). También son propensos a desarrollar laminitis así como otros problemas, incluyendo infertilidad de las yeguas, anhidrosis y rabdomiólisis al esfuerzo (Breuhaus \& LaFevers, 2005). En las yeguas que se identificó una ingestión excesiva de yodo se observó bradicardia, obesidad y letargia, al igual que edema en extremidades traseras, capa de pelo gruesa y una disminución en el apetito (Toribio \& Duckett, 2004). Se han descrito casos de alopecia atribuida a un exceso de yodo y bajos niveles de TH circulantes; un caso describe agalactia asociada a una disminución de la función tiroidea (Glover et al., 2009). Es fundamental resaltar el hecho de que tanto el hipotiroidismo como el hipertiroidismo tienen como una de sus consecuencias la disminución en el nivel de testosterona circulante (McLaughlin, Doige \& McLaughlin, 1986), es natural asumir que los procesos dependientes de dichas hormonas se van a ver afectados, siendo unos de los más seriamente disminuidos la menor movilidad del esperma eyaculado y la menor maduración del espermatozoide en el epidídimo (Nicassio et al., 2008). La disminución de la toma de yodo es considerada la mayor causa de hipotiroidismo neonatal y bocio, por ello el tamaño de la glándula tiroides en potros puede ser normal o aumentado (McLaughlin \& Doige, 1982). La disminución de las concentraciones de TH estimula la secreción de TSH por parte de la glándula pituitaria, la cual a su vez estimula las células tiroidales resultando en un aumento de la glándula tiroides (Frank, 2009; Baragli et al., 2011). La ingestión diaria de yodo en adultos es de $0,007 \mathrm{mg} / \mathrm{kg}$ o de $0,35 \mathrm{mg} / \mathrm{kg}$ de materia seca en la dieta, pudiéndose incrementar a 0,4 $\mathrm{mg} / \mathrm{kg}$ de materia seca para el tercer último trimestre de gestación (Breuhaus \& LaFevers, 2005). La mayoría de los signos clínicos reportados en hipotiroidismo de potros se asocia al rol crucial de las $\mathrm{TH}$ en 
el desarrollo y maduración de los sistemas nervioso y musculoesquelético (McLaughlin \& Doige, 1982; Toribio \& Duckett, 2004). Las TH tienen efectos mayores en el desarrollo del cerebro durante el periodo pre y postnatal y la deficiencia de $\mathrm{TH}$ resulta en una disminución del crecimiento axonal, arborización dendrítica y mielinización de áreas del sistema nervioso central (Toribio \& Duckett, 2004). Las hormonas tiroideas son muy importantes en el desarrollo del cerebro del neonato, por ello un suministro de T4 como tratamiento temprano previene el daño intelectual y es la mayor razón de alarma en el daño de la función tiroidea en los neonatos humanos (Toribio \& Duckett, 2004). El hipotiroidismo en los potros algunas veces se manifiesta desde el nacimiento, donde la posición no es la adecuada, y presentan un reflejo de succión pobre o nulo (Barsnick \& Toribio, 2011). Se presenta un retraso en la osificación de los huesos principalmente del carpo cubital, presentan disgenesia fiseal, debilidad, incoordinación y muerte. Presentan defectos en la osificación con colapso de hueso tarsal, hipoplasia u osificación incompleta del tercer y cuarto hueso del carpo, osteocondritis disecante del tercer y cuarto hueso carpal, hipoplasia y colapso del hueso tercero y central del tarso (Beech, 1998; Messer et al., 1995), huesos carpianos hipoplásicos, ruptura del extensor digital común, contracción de miembros anteriores, y prognatismo mandibular, largo pelo hirsuto e insuficiencia respiratoria (Allen et al., 1996). Los potros pueden nacer aparentemente normales pero desarrollan lesiones esqueléticas semanas después (Toribio \& Duckett, 2004). La tiroxina juega un papel en la maduración de los pulmones y en la producción del surfactante, lo cual se desencadena en problemas respiratorios como muerte por asfixia al nacimiento (Toribio \& Duckett, 2004).

\section{DIAGNÓSTICO}

Las pruebas tiroideas pueden clasificarse de acuerdo a la información que proveen las pruebas sobre función, etiología y anatomía. Las pruebas son directas cuando se miden las concentraciones de TH y TRH en suero e indirectas cuando se calculan las concentraciones de $\mathrm{TH}$ por modelos matemáticos 0 índices, o cuando se determina la relación entre otros metabolitos y la función tiroidea (Toribio \& Duckett, 2004). Las concentraciones en suero de T4L medida por diálisis es el método más certero para diagnosticar la enfermedad tiroidea, comparado con otros métodos de medición de T4L (Breuhaus \& LaFevers, 2005). La medición de yodo en suero no es certera como prueba diagnóstica ya que las concentraciones de yodo en la glándula tiroides son 30-40 veces mayores que las encontradas en el suero (Nicassio et al., 2008). El yodo es transportado de forma activa al interior de los folículos tiroideos y comienza a fijarse después de la oxidación (Medica et al., 2011). Las concentraciones de yodo pueden estar disminuidas incluso si las concentraciones en sangre son normales (Frank, Sojka \& Messer, 2002). 
TSH: La determinación de los niveles de TSH en suero se considera estratégica para evaluar la funcionalidad de la glándula tiroides, ya que es muy eficiente y de un costo favorable (Toribio \& Duckett, 2004). Para identificar el hipotiroidismo primario de otras alteraciones del eje HPT, se deben medir las concentraciones de TSH. Unos niveles de T3T, T4T y T4L bajos son acompañados de unas concentraciones elevadas de TSH, la secreción de TSH incrementa para estimular la síntesis de TH cuando la alteración se encuentra en la glándula (Frank, Sojka \& Messer, 2002).

T4: Se miden las concentraciones de esta hormona por medio de inmunoensayos y se mide tanto la T4L como la T4T o ligada a proteínas (Toribio \& Duckett, 2004). Animales que al examen reporten niveles que sean inferiores a los $1,5 \mathrm{mg} / \mathrm{dl}$ son hipotiroideos (Abraham et al., 2011).

T3: De igual forma son más precisos los métodos utilizados para medir los niveles séricos de las THs libres, ya que siempre y cuando se encuentren ligadas a proteína (albúmina, prealbúmina) se van a observar resultados modificados por el metabolismo proteico precedente a la toma de la muestra (Toribio \& Duckett, 2004).

Pruebas indirectas: Las pruebas de estimulación proveen una información más precisa del estado tiroideo que las mediciones solas de las concentraciones de tiroides, estas pruebas incluyen las mediciones de las concentraciones de hormona tiroidea antes y después de la aplicación de TRH o TSH (hormona estimulante tiroidea) para estimular la producción de TH (Breuhaus, 2011).

Otras pruebas de laboratorio: El colesterol en suero es elevado en el hipotiroidismo primario en humanos y perros, pero no es una respuesta específica en la disfunción tiroidea. El incremento en las concentraciones de colesterol se ha encontrado en algunos caballos con hipotiroidismo pero en otros no, entonces no se considera un indicador válido en equinos (Toribio \& Duckett, 2004). También se ha identificado un aumento en las concentraciones de triglicéridos y lipoproteínas de baja densidad (Breuhaus \& LaFevers, 2005). Cuando ya la enfermedad es crónica se evidencia una anemia normocrómica, aunque no es específica puede observarse en hipotiroidismo (Toribio \& Duckett, 2004). Cuando el animal presenta ataque de mioglobinuria y azoturia después de haber desarrollado ejercicio físico, los niveles de AST y CK (aspartato aminotransferasa y creatinkinasa) aumentan en la sangre y en un caballo hipotiroideo estas enzimas únicamente retornan a sus valores basales cuando se suministra la hormona por vía oral (Geor, 2010).

Prueba de respuesta trófica: Es utilizada para diferenciar la disfunción primaria o secundaria de la glándula tiroides y no se presentan las variaciones por factores endógenos o exógenos que afectan las otras pruebas (Toribio \& Duckett, 2004). 
TSH: El protocolo de la TSH consiste en la inyección de 5 UI de TSH intravenosa y se comparan niveles de TH antes y después del suministro. En los caballos eutiroideos los picos de T4 se dan de 3 a 4 horas posinyección a una concentración 2,4 veces mayor que los niveles preinyección (Walsh et al., 2009). La T3 se duplica 30 minutos luego del suministro con un pico a las 2 horas con una concentración 5 veces mayor a la basal (Divers, 2008). La respuesta normal se caracteriza por un aumento de T3 que antecede el aumento de T4 el cual es mayor. Cuando se realiza la aplicación de las 5 UI de TSH por vía intramuscular los picos de T4 son 2 veces por encima del nivel basal 3 a 12 horas después de inyección y los picos de T3 se observan de 1 a 3 horas de la aplicación. El protocolo sugiere que no existe diferencia entre la aplicación de 2,3 UI y 5 UI por vía intramuscular, pero se deben medir los niveles de las hormonas de 3 a 6 horas posteriores al suministro (Breuhaus, 2011). Cuando se analizan los niveles de tiroxina se puede diagnosticar la presencia de disfunción primaria de la tiroides ya que un deficiente incremento de tiroxina es un indicador de esta alteración (Toribio \& Duckett, 2004). La administración de fenilbutazona en los caballos disminuye las concentraciones de las hormonas tiroideas pero no afecta la prueba de estimulación de TSH. La dexametasona disminuye las TH en la prueba de respuesta de TSH (Toribio \& Duckett, 2004). En un paciente con hipotiroidismo una aplicación de 5 Ul de TSH por vía intravenosa resultará en un aumento (mayor a dos veces) de T3 y T4 en suero después de 2 a 6 horas luego de la administración. Infortunadamente tanto la medición de TSH endógena como la TSH exógena no son muy viables debido a la incapacidad de los laboratorios para ofrecer las pruebas ya que no se ha podido establecer una consistencia en los ensayos (Frank, Sojka \& Messer, 2002).

TRH: El test de estimulación de TRH consiste en una administración de TRH vía intravenosa de $1 \mathrm{mg}$ para caballos y $0,5 \mathrm{mg}$ para ponis (Messer et al., 1995). Una inadecuada respuesta de TRH puede ocurrir en el hipotiroidismo primario o secundario. En animales normales los picos de T4 ocurren a las 4 h de la aplicación y de T3 a las 2 h (Tahboub \& Arafah, 2009). En otro estudio se encontró que T3 se incrementa significativamente de 1 a 2 h después de la aplicación con picos a las 2-4 horas. La tiroxina incrementa por 2 horas y logra el pico a las 4-10 horas luego de la inyección de TRH (Divers, 2008). La aplicación de TRH presenta efectos colaterales como son salivación, vómito, contracción pupilar, taquicardia, taquipnea, relajación de esfínter anal y urinario (Himler et al., 2010). Si se observa una respuesta baja de TRH sugiere disfunción a nivel de la glándula pituitaria (Divers, 2008). Las concentraciones basales de TH bajas con concentraciones altas de TSH y una respuesta anormal (baja) al test de estimulación de TSH sugieren disfunción de la glándula tiroides. Bajos niveles de TH basales con una concentración normal o baja de TSH sugieren disfunción de la glándula pituitaria o del hipotálamo. Bajos niveles de TH y TSH basales con respuesta al test de TRH pueden sugerir disfunción hipotalámica como causa de hipotiroidismo. El test de estimulación de TRH ha sido 
usado para diagnosticar adenomas pituitarios en caballos (Toribio \& Duckett, 2004).

Prueba de supresión THs: Por medio de este test se puede demostrar la presencia de TSH autónoma o independiente de la función de la glándula tiroides. La administración exógena de $\mathrm{TH}$ en cantidades suficientes para suprimir las concentraciones de TSH resulta en una disminución de la síntesis y secreción de THs. La supresión de hormona tiroidea indica si la función de la glándula tiroides es independiente de TSH. La administración de T3 (prueba de supresión de T3) para individuos saludables se realiza para causar una disminución de las concentraciones de T4 en suero, sin embargo algunos animales no presentan una disminución significativa de T4 luego de la administración de T3. Esta prueba fue usada para diagnosticar hipertiroidismo en un caballo con adenoma tiroideo; 2,5 mg de T3 se suministraron por vía intramuscular y un examen simple reveló que no hubo una disminución en las concentraciones de T4 (Himler et al., 2010).

Diagnóstico de hipotiroidismo en potros: Principalmente se evalúan signos anteriormente mencionados con o sin aumento del tamaño de la glándula tiroides. Se puede examinar a la yegua y determinar su estado nutricional. El aumento de la glándula tiroides no es precisamente un indicador de hipo o hipertiroidismo, se debe evaluar la función por medio de la prueba para confirmar el diagnóstico. Un punto crucial para recordar es que los altos niveles de concentración de THs son normales en potros (Douglas, 1999). No solo una unidad central poderosa existe para estimular el eje tiroideo durante la gestación tardía, sino que también existen concentraciones de globulinas ligadoras de tiroxina que incrementan la tiroxina en los neonatos. Las concentraciones de T4T, T4L, T3T y T3L en la sangre del cordón umbilical del potro recién nacido son de $14,5,7$ y 3 veces mayores respectivamente, comparadas con las concentraciones en caballos adultos (Messer \& Johnson, 2007). Para la mayoría de los fetos la T4 es sin yodo a rT3, y las concentraciones de T3 son bajas mientras que las concentraciones de T4 y rT3 son altas. Al nacimiento la producción de rT3 disminuye, y la desyodación deja T3. Las concentraciones en plasma de rT3 en potros disminuyen rápidamente al mismo tiempo que aumenta la T3 las primeras 24 a 48 horas de vida, se presentan mesetas los próximos 2 a 3 días donde T4 declina de forma paralela (Toribio \& Duckett, 2004). Una disminución en las concentraciones de T4 ocurre durante los primeros 16 días de vida. La T4L disminuye durante los 3 primeros meses de vida. Las concentraciones de T3 en suero son elevadas por encima de la de adultos las primeras 6 a 12 horas, y en 24 horas declina significativamente (Douglas, 1999) Cuando se evalúan las concentraciones de TH en potros que tienen hipotiroidismo, se deben comparar con las concentraciones de THs de los potros saludables de la misma edad. Un potro puede evidenciar hipotiroidismo pero las THs pueden estar dentro del rango, sugiriendo que tal vez una deficiencia de THs ocurre durante el desarrollo (Toribio \& Duckett, 2004). 


\section{FACTORES QUE ALTERAN LAS PRUEBAS DIAGNÓSTICAS}

Existen ciertos factores que alteran los resultados de los test diagnósticos, lo cual se debe tener en cuenta para evaluar un paciente con posible alteración de la glándula tiroides (Himler et al., 2010). Uno de estos factores es la edad; un estudio reciente encontró que las concentraciones de T3 en plasma disminuían $7,9 \mathrm{ng} / \mathrm{ml}$ desde el nacimiento a $0,9 \mathrm{ng} / \mathrm{ml}$ a los 6 meses de edad y a $0,7 \mathrm{ng} / \mathrm{ml}$ a los 9 meses de edad. De igual forma las concentraciones de T4 disminuyeron de $233 \mathrm{ng} / \mathrm{ml}$ al nacimiento a $49 \mathrm{ng} / \mathrm{ml}$ a los 14 días y a $35 \mathrm{ng} / \mathrm{ml}$ a los 6 meses (Douglas, 1999). Las diferencias en las concentraciones de THs en los géneros no son significativas, sin embargo si se hallan diferencias entre los estadios reproductivos de las hembras donde aquellas yeguas que se encontraban en gestación presentaban niveles superiores de THs, se cree que probablemente sea por un aumento de las concentraciones de TBG durante el periodo gestante (Messer \& Johnson, 2007). De igual forma, las concentraciones de tiroxina son significativamente mayores en las hembras entre los días 49 y 55 de gestación comparado con otras hembras en gestación avanzada, sin embargo la T3 no varía en las hembras en periodo gestante (Frank et al., 1999 Boosinger et al., 1995). Algunas sustancias disminuyen el funcionamiento de las hormonas tiroideas como son: los glucocorticoides, los andrógenos, salicilatos, otros antinflamatorios no esteroideos como fenilbutazona y agentes quimioterapéuticos (Abraham et al., 2011). Los glucocorticoides inhiben la secreción endógena de TRH y disminuyen la respuesta de la TSH a la TRH. Los glucocorticoides también pueden inhibir la desyodación periférica en los adultos. En neonatos mejoran la conversión de T4 a T3. De igual forma disminuyen la TBG, aumentan la TBP, disminuyen la T4T, e incrementan la T4L (Toribio, 2011). Las catecolaminas aumentan la tasa de desyodación de T4 a T3 y tienen un efecto potenciador (Furr, Murray \& Ferguson, 1992). Los estrógenos aumentan la producción de TBG y la androgénesis tiende a suprimirla (Geor, 2008; Himler et al., 2010). La deficiencia de selenio y las situaciones de estrés también afectan de manera notable las concentraciones de THs (Berger et al., 2001). En muchos caballos eutiroideos también se pueden observar bajos niveles séricos de T4 acompañados de resistencia a la insulina y de hiperinsulinemia (Frank et al., 2005). En una investigación se observó que luego de una alimentación con un alto porcentaje de energía y de proteína en los potros destetos de 6 a 8 meses de edad la conversión periférica de T4 a T3 resultó en una disminución relativa de T4 3 horas posteriores a la alimentación (Messer \& Johnson, 2007). La acelerada conversión de T4 a T3 luego de la alimentación rica en carbohidratos se relaciona con el incremento en la secreción de insulina debido al aumento de glucosa, ya que la insulina acelera la desyodación de T3 (Furr, Murray \& Ferguson, 1992). En los equinos la existencia de biorritmos en la secreción de las hormonas también sigue siendo estudiada en la actualidad, situación que está directamente influenciada por la actividad que desarrollan los 
animales (sedentarios, de trabajo, de competencia); por ende, es muy importante dilucidar si existen o no cambios circadianos significativos en los niveles hormonales cuando los caballos sedentarios empiezan una rutina de entrenamiento (Medica et al., 2011), investigaciones anteriores han reportado el cambio estacional de yodotironinas (Christensen et al., 1997; Fazio et al., 2007). Se han reportado variaciones a lo largo del día de las iodotironinas (Duckett et al., 1989), sin embargo esta situación no ha sido bien aclarada pues son muchos los factores que alteran los ciclos hormonales de un caballo, sobre todo cuando este se encuentra sometido a un régimen de ejercicio (Baragli et al., 2008), al estrés propio de los cambios de comida, los desplazamientos, los cambios de pesebrera y demás factores relacionados con el mundo de las competiciones equinas (Fazio et al., 2007; Medica et al., 2011). Cuando se comparan los niveles tiroideos en caballos cuarto de milla que han recibido un entrenamiento previo con aquellos que no la han recibido, se nota claramente el cambio de la cantidad de iodotironinas y sus patrones durante un período de 24 horas (Fazio et al., 2007). Aún no es clara la razón por la cual aumentan los niveles de las hormonas en la sangre, dentro de las posibilidades que se consideran están: los cambios en la capacidad de unión a las proteínas por parte de las iodotironinas para su posterior eliminación o a un aumento de la secreción de hormonas por parte de la glándula (Furr, Murray \& Ferguson, 1992). En los caballos que no habían tenido entrenamiento previo se reportaron índices hormonales cuyos niveles aumentaban durante la noche, mientras que en los caballos que habían tenido un entrenamiento previo los niveles más altos de las hormonas en la sangre fueron encontrados en las horas postmeridiano (Baragli et al., 2008; Medica et al., 2011). Por lo tanto, el entrenamiento parece generar un efecto en el queT4L se hace más disponible en el cuerpo del animal y su pico máximo en la sangre se adelanta aproximadamente unas 4 horas (Medica et al., 2011). Es importante recalcar la estación del año en la cual se miden los niveles de THs ya que si es una estación fría se van a encontrar resultados incrementados (Hulbert, 2000). Investigaciones recientes concluyen que cuando hay inanición se produce una disminución mayor al 50\% de T3 y T4 en suero en solo 1 a 2 días (Toribio, 2011). Entre las sustancias que tienen efectos bociógenos por bloqueo o competencia por captación del yodo se encuentra el tiocianato y el perclorato. La oxidación del yodo y la captación de yodotiroxinas son inhibidas por drogas como son las sulfonamidas, fenilbutazona, fenotiazinas, tiouracilo, tiopental y el metimazol. Los alimentos a base de soya, linaza y plantas del género Brassica contienen sustancias bociógenas (Hulbert, 2000). La administración de fenilbutazona disminuye las concentraciones de $\mathrm{TH}$ en los caballos, y este efecto puede durar hasta 10 días posteriores a la administración, por lo tanto los niveles de TSH en las pruebas pueden encontrarse normales o exacerbados en los caballos tratados con fenilbutazona (Murray, 1990). De igual forma, los glucocorticoides disminuyen los niveles de TSH y TRH, disminuyendo la T3 y la T4 en suero. Un estudio reciente demostró que los caballos saludables tratados con dexametasona durante 5 días, incrementaron la rT3 en el suero 
y T3L (Toribio \& Duckett, 2004). Altos niveles de nitratos en el agua pueden resultar en una hipertrofia de la glándula tiroides, además que reducen el transporte activo del yodo en la glándula tiroides (Ferguson, 1988). El propiltiouracilo afecta el metabolismo del yodo inhibiendo la conversión periférica de T4 a T3 (Murray, 1990). El fenobarbital incrementa la TH ligada a los microsomas hepáticos e incrementa la desyodación. La reserpina, agente depresor de catecolaminas, inhibe la secreción de TSH e inhibe la desyodación. La antipirina tiene una actividad antitiroidea. Esteroides anabólicos pueden tener un efecto inhibitorio sobre la TBG (Toribio \& Duckett, 2004). Los niveles de yodo pueden interferir sobre los test de medición de hormonas tiroideas. Los caballos pueden estar en contacto con sustancias yodadas como son comidas ricas en yodo, expectorantes, pinturas para las patas, champús, inyecciones irritantes, radiografías con medio de contraste y drogas antiprotozoarias (Ferguson, 1988).

El ejercicio induce cambios en las concentraciones de las hormonas plasmáticas; los cambios en las concentraciones de las hormonas activas libres, son menos afectados que las hormonas ligadas a proteínas. Es por ello que la medición de hormonas debe realizarse en estado de reposo, y se debe tener en cuenta si el caballo es atlético o no (Hyypp, 2005).

\section{SÍNDROME DE ENFERMEDAD NO TIROIDEA}

Se denomina síndrome de enfermedad no tiroidea (NTES, por sus siglas en inglés) cuando existen bajas concentraciones en los niveles de T3 mientras que la T4 puede estar baja, normal o elevada (De Groot, 1999; De Groot, 2006). Las concentraciones de $\mathrm{TH}$ como es sabido disminuyen por inanición y por enfermedad. Primero las concentraciones de T3 disminuyen y en la severidad de la enfermedad disminuyen las concentraciones de T4 también (Christensen et al., 1997). La inanición resulta en una disminución de T3 y T3L. La privación de los carbohidratos inhibe la desyodación de T4 a T3 por la desyodinasa tipo I, lo cual resulta en unas concentraciones bajas de T3 y un incremento en las concentraciones de rT3 (De Groot, 2006). Esto ocurre porque el metabolismo basal disminuye como respuesta adaptativa del organismo para conservar calorías induciendo a un estado hipotiroideo (Hulbert, 2000). Estudios recientes han encontrado que la causa más probable del NTES es la disfunción a nivel hipotalámico y de la glándula pituitaria. Se ha detectado una disminución en la expresión del fRNA de la TRH a nivel hipotalámico al presentarse inanición en ratas y en pacientes humanos con NTES (Warner \& Beckett, 2010). Esta teoría se comprueba cuando al suministrar TRH a estos pacientes se incrementa TSH, T4 y T3 (Fitzgerald \& Davidson, 1998). En la patogenia del NTES se encuentran como principales responsables las citoquinas, los glucocorticoides y más recientemente la leptina. Los glucocorticoides juegan un papel muy importante ya que suprimen la secreción de TSH, lo cual se puede desencadenar 
en distintas condiciones como son estrés, sepsis o disfunción de la glándula pituitaria (síndrome de Cushing) (Christensen et al., 1997). A pesar de que el NTES es un estado hipotiroideo, la evidencia sugiere que el daño a los tejidos químicamente es mínimo y que la disminución en las concentraciones de TH son reconocidas como benéficas, sin embargo un proceso de mala adaptación ocurre en algunos casos con un baja en las concentraciones de T4 lo cual se ha asociado a un incremento de la mortalidad (Geor, 2008). Se ha sugerido que el suministro de TH exógeno para la terapia del NTES puede ser más perjudicial ya que logra inhibir el eje HPT por retroalimentación negativa, evitando por consiguiente la producción endógena de la misma (Toribio \& Duckett, 2004).

\section{PREVENCIÓN Y TRATAMIENTO}

Para el tratamiento de los caballos que realizan ejercicio y sufren de un ataque después de haber desarrollado un esfuerzo, se recomienda la administración de $10 \mathrm{mg}$ de levotiroxina sódica diaria hasta que la signología mejore (Fazio et al., 2007). Cuando el diagnóstico es por bajos niveles en las concentraciones de THs o por bocio hiperplásico, se debe verificar el suministro de yodo en la dieta (Murray, 1990). Luego se debe diferenciar el hipotiroidismo de la enfermedad no tiroidea ya que en el último caso no se hará tratamiento por hipofunción, ya que podría perjudicarlo produciendo una inhibición del eje HPT y suprimir la síntesis y secreción endógena de THs (Toribio, 2011). El Consejo Nacional de Investigación recomienda un mínimo de yodo diario de 0,1 $\mathrm{mg} / \mathrm{kg}$ en la dieta, un total de 1-2 $\mathrm{mg}$ de yodo al día por animal ha sido reportado. La caseína yodada o tirotropina a $5 \mathrm{mg}$ por día reversa los efectos en los caballos tiroidectomizados, y a 5$10 \mathrm{mg}$ oral por día, reversa la anemia asociada a la tiroides y la miopatía en los caballos de carreras (Breuhaus \& LaFevers, 2005). Extracto de tiroides disecada a $2 \mathrm{mg} / \mathrm{kg}$ de masa corporal suministrado vía oral una vez al día ha demostrado ser exitoso (Toribio \& Duckett, 2004). La suplementación con T4 puede ser efectiva solo si hay un defecto en la desyodación. Se comienza con una dosis de $20 \mathrm{mcg}$ de T4 por $\mathrm{kg}$ de masa corporal al día. Si se suplementa con T3 se debe suministrar 1 $\mathrm{mg} / \mathrm{kg}$ al día (Murray, 1990). Sin embargo, el suplemento de hormonas tiroideas aún presenta muchas controversias y requiere una larga escala de estudios (Breuhaus \& LaFevers, 2005). Se debe monitorear al animal luego de 15 días de iniciar la terapia, midiendo los niveles de hormonas tiroideas periódicamente (Breuhaus \& LaFevers, 2005). El tratamiento médico a partir de la levotiroxina es una de las opciones para manejar a los caballos obesos o insulinorresistentes que no responden al tratamiento conservativo, el cual consiste en disminuir el consumo de calorías y aumentar la actividad física para perder peso y mejorar la sensibilidad de los tejidos diana a la insulina. Todo esto tiene como fin último evitar que el animal desencadene un cuadro de laminitis (Breuhaus \& LaFevers, 2005). Cuando el metabolismo de las hormonas tiroideas se ha reducido, puede ser estimulado a través de la aplicación de 
estrógenos exógenos los cuales hacen que la captación de yodo por parte de la tiroides y de los ovarios aumente así como aumentar la utilización en los tejidos periféricos de hormonas tiroideas (Geor, 2008). Caballos obesos que sufren de laminitis han alcanzado mejoría clínica después de la administración de suplementos tiroideos (Geor, 2008; Walsh et al., 2009). La levotiroxina sódica puede suministrarse a las caballos que sufren de SME con el fin de reducir su peso y aumentar la sensibilidad a la acción de la insulina (autógena). Estos resultados fueron obtenidos cuando la levotiroxina sódica fue aplicada en yeguas saludables a unas dosis que variaba entre los 24 y 96 mg/día durante 8 semanas (Frank et al., 2005). Los efectos a largo plazo de la levotiroxina fueron evaluados en yeguas sanas por un período mayor a 48 semanas (Durham, 2010). Los resultados arrojados después de las 48 semanas, de

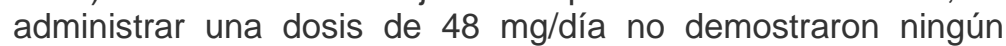
efecto adverso (Durham, 2010). Debe tenerse en cuenta que cuando un animal está en tratamiento con este medicamento, su dieta debe ser monitoreada de manera estricta pues la pérdida de peso se dificulta cuando el animal puede pastar libremente. Las concentraciones séricas de T4 varían notablemente durante el tratamiento dependiendo de la dosis de medicamento suministrada, el animal y la hora del día en la que la muestra es tomada; por lo general los resultados de T4 séricos para caballos en tratamiento se encuentran entre los 40 y $100 \mathrm{ng} / \mathrm{ml}$, resultados indicadores de una administración supra fisiológica del medicamento, sin embargo signos clínicos de hipertiroidismo tales como la taquicardia no fueron vistos en los caballos tratados (Hurcombe, 2011). No se han identificado efectos adversos en los caballos tratados con levotiroxina excepto por agitación las dos últimas semanas de estudio de un periodo de 8 semanas. Se observó un aumento en el ritmo cardiaco como única anomalía (Frank et al., 2005). La administración de T4L reduce significativamente el peso, disminuye las concentraciones de lipoproteínas de baja densidad (VLDL), triglicéridos totales (TG) y colesterol total (TC) y altera la dinámica de la glucosa en los caballos adultos (Frank et al., 2005). Cuando el hipotiroidismo es secundario la suplementación con hormona tiroidea solo debe ser considerada cuando se identifican bajos niveles de THs, se observan signos clínicos de hipotiroidismo, y se han descartado posibles agentes que alteren la función tiroidea (Durham, 2010). La prevención se basa especialmente en los casos de hipotiroidismo en potros, donde se orienta a una dieta que cumpla con todos los requerimientos según la NRC (National Research Council) (Nicassio et al., 2008). El neonato obtiene los minerales de las reservas corporales de la madre mediante la leche, lo cual depende del suplemento de los mismos en la dieta de la yegua ya sea en forrajes o concentrados (Kavazis, Kivipelto \& Ott, 2002). Cuando las yeguas son suplementadas con trazas de minerales y altos niveles de minerales como son el cobre, yodo, zinc y hierro, según la NRC, existe una menor posibilidad de que el potro desarrolle alteraciones como debilidad al nacer y anormalidades óseas, y aunque no existen estudios precisos sobre la suplementación con yodo y el hipotiroidismo en potros este estudio general indica que cuando se suministran minerales a la yegua durante el último periodo 
de gestación se garantiza un potro en mejores condiciones para enfrentar los primeros días de vida (Kavazis, Kivipelto \& Ott, 2002).

\section{CONCLUSIONES}

El hipotiroidismo una de las alteraciones endocrinas más comunes en los equinos, afectando poblaciones desde temprana edad, denominándose con el nombre de cretinismo en potros a la deficiencia de hormonas tiroideas. Los signos clínicos en los potros son muy característicos incluyendo letargia, retraso en el crecimiento, debilidad e incoordinación, hasta llegar finalmente a la muerte. Es de fundamental importancia la nutrición de la yegua durante el periodo de gestación, ya que la deficiencia en minerales afecta notablemente el desarrollo del feto y los niveles de hormonas tiroideas circulantes del potro en sus primeros días de vida. El hipotiroidismo puede deberse a enfermedades adyacentes que afectan la función tiroidea al igual que componentes exógenos que afectan el tejido tisular e interfieren con la síntesis de hormonas tiroideas. El método diagnóstico más certero para diagnosticar enfermedad tiroidea es la medición de las concentraciones de T4L en suero por diálisis, mientras que la medición de yodo en suero no es certera como prueba diagnóstica ya que las concentraciones de yodo en la glándula tiroides son 30-40 veces mayores que las encontradas en el suero. La levotiroxina sódica continúa siendo el tratamiento de elección para el hipotiroidismo.

Tabla 1. Valores de hormonas tiroideas en equinos

\begin{tabular}{|l|c|c|c|c|l|}
\hline \multicolumn{1}{|c|}{ Estado } & $\begin{array}{c}\text { T4T } \\
(\mathbf{n M})\end{array}$ & $\begin{array}{c}\text { T3T } \\
(\mathbf{n M})\end{array}$ & $\begin{array}{c}\text { T4L } \\
(\mathrm{pM})\end{array}$ & $\begin{array}{l}\text { T3L } \\
\text { (pM) }\end{array}$ & Referencia \\
\hline En reposo & 8,6 & 0,7 & & $\begin{array}{l}\text { Messer et al. } \\
(1998)\end{array}$ \\
\hline Agitado & 9,0 & 1,0 & & $\begin{array}{l}\text { Irvine \& Evans } \\
(1975)\end{array}$ \\
\hline Adulto & 19,9 & 1,0 & 11,6 & 2,1 & $\begin{array}{l}\text { Messer et al. } \\
(1995)\end{array}$ \\
\hline Adulto & 34,8 & 1,2 & 33,2 & 8,8 & $\begin{array}{l}\text { Irvine \& Evans } \\
(1975)\end{array}$ \\
\hline
\end{tabular}

\section{BIBLIOGRAFÍA}

- Abraham, G.; Allersmeier, M.; Schusser, G.F. \& Ungemach, F.R. (2011). Serum thyroid hormone, insulin, glucose, triglycerides and protein concentrations in normal horses: Association with topical dexamethasone usage. Vet J, 188, 307-312.

- Ahmed, O.M., El-Gareib, A.W.; El-Bakry, A.M.; Abd ElTawab, S.M. \& Ahmed, R.G. (2008). Thyroid hormones 
states and brain development interactions. Int. J. Devl Neuroscience, 26, 147-209.

- Allen, A.L.; Townsend, H.G.; Doige, C.E. \& Fretz, P.B. (1996). A case-control study of the congenital hypothyroidism and dysmaturity syndrome of foals. Can Vet J, 37, 349-358.

- Baragli, P.; Sgorbini, M.; Casini, L.; Ducci, M. \& Sighieri, C. (2011). Early Evidence of the Anticipatory Response of Plasma Catecholamine in Equine Exercise. Equine Vet J, 31, 85-88.

- Barsnick, R.J. ; Toribio, R.E. (2011). Endocrinology of the equine neonate energy metabolism in health and critical illness. Vet Clin Equine, 27, 49-58.

- Beech, J. (1998). Disorder s of thyroid gland function. In: Watson, T. (ed.). Metabolic and endocrine problems of the horse (pp. 69-74). Philadelphia: WB Saunders.

- Berger, M.M.; Reymond, M.J.; Shenkin, A. et al. (2001). Influence of selenium supplements on the posttraumatic alterations of the thyroid axis: a placebocontrolled trial. Intensive Care Med, 27, 91-100.

- Boosinger, T.R.; Brendemuehl, J.P.; Bransby, D.L. et al. (1995). Prolonged gestation, decreased triiodothyronine concentration, and thyroid gland histomorphologic features in newborn foals of mares grazing Acremonion coenophialum-infected fescue. Am J Vet Res, 56, 66-9.

- Breuhaus, B.A. (2011). Disorders of the Equine Thyroid Gland. Vet Clin North Am Equine Pract, 27(1), 115-128.

- Breuhaus, B.A. \& LaFevers, D.H. (2005). Thyroid function in normal, sick and premature Foals [abstract]. $\mathrm{J}$ Vet Intern Med, 19, 445.

- Christensen, R.A.; Malinowski, K.; Massenzio, A.M.; Hafs, H.D. \& Scanes, CG. (1997). Acute effects of short-term feed deprivation and refeeding on circulating concentrations of metabolites, insulin-like growth factor $i$, insulin-like growth factor binding proteins, somatotropin, and thyroid hormones in adult geldings. $J$ Anim Sci, 75, 1351-1358.

- De Groot, L.J. (1999). Dangerous dogmas in medicine: the nonthyroidal illness syndrome. J Clin Endocrinol Metab, 84, 151-64.

- De Groot LJ. (2006). Non-thyroidal illness syndrome is a manifestation of hypothalamicpituitary dysfunction, and in view of current evidence, should be treated with appropriate replacement therapies. Crit Care Clin, 22, 57-86.

- Divers, J.T. (2008). Endocrine Testing in Horses: Metabolic Syndrome and Cushing's Disease. Equine Vet J, 28(5), 315-316.

- Douglas, R. (1999). Circadian cortisol rhythmicity and equine cushing's-like disease. Equine Vet J, 19(11), 684, 750-751, 753.

- Durham, A. (2010). The Pharmacologic Basis for the Treatment of Endocrinopathic Laminitis. Vet Clin North Am Equine Pract, 26(1), 115-128.

- Duckett WM. (1989). Thyroid gland. Equine internal medicine. 917-923. 
- Evans MJ, Irvine CH. (1975). Serum concentrations of $\mathrm{FSH}, \mathrm{LH}$ and progesterone during the oestrous cycle and early pregnancy in the mare. Journal of Reproduction and Fertility. 23:193-200.

- Fazio, E.; Medica, P.; Cravana, C.; Messineo, C. Ferlazzo, A. (2007). Total and free iodothyronine levels of growing Thoroughbred foals: Effects of weaning and gender. Livestock Science, 110(3), 207-213.

- Ferguson, D.C. (1988). The effect of nonthyroidal factors on thyroid function tests in dogs. Comp Contin Educ Pract Vet, 10(12), 1365-77.

- Fitzgerald, B.P. \& Davison, LA. (1998). Thyroxine concentrations are elevated in mares which continue to exhibit estrous cycles during the nonbreeding season. Equine Vet J, 18 (1), 48-51.

- Frank, N. (2009). Equine Metabolic Syndrome. Vet Clin North Am Equine Pract, 29(5), 239-255.

- Frank, N.; Sojka, J.E.; Latour, M.A. et al. (1999). Effect of Hypothyroidism on blood lipid concentrations in horses. Am J vet Res, 60, 730-733.

- Frank, N.; Sojka, J. \& Messer, N.T. (2002). Equine thyroid dysfunction. Vet Clin Equine, 18, 305-319.

- Frank, N.; Sommardah, C.S.; Eiler, H.; Webb, L.L. \& Denhart, J.W. (2005). Effects of oral administration of levothyroxine sodium on concentrations of plasma lipids, concentration and composition of very-lowdensity lipoproteins, and glucose dynamics in healthy adult mares. Am J Vet Res, 66(6), 1032-1038.

- Furr, M.O.; Murray, M.J. \& Ferguson, D.C. (1992). The effects of stress on gastric ulceration, T3, T4, reverse T3 and cortisol in neonatal foals. Equine Vet J, 24, 3740.

- Geor, R.J. (2008). Metabolic Predispositions to Laminitis in Horses and Ponies: Obesity, Insulin Resistance and Metabolic Syndromes. Equine Vet J, 28(12), 753-759.

- Geor, R.J. (2010). Nutrition and Exercise in the Management of Horses and Ponies at High Risk for Laminitis. Equine Vet J, 30(9), 463-470.

- Glover, C.M.; Miller, L.M.; Dybdal, N.O.; López, A.; Duckett, W.M. \& McFarlane, D. (2009). Extrapituitary and Pituitary Pathological Findings in Horses with Pituitary Pars Intermedia Dysfunction: A Retrospective Study. Equine Vet J, 29(3), 146-153.

- Himler, M.; Cattaneo, A.; Barsnick R.J. et al. (2010). Thyroid hormones in healthy, sick nonseptic and septic foals [Research Abstract Program of the 2010 ACVIM Forum]. J Vet Intern Med, 24, 784.

- Hulbert, A.J. (2000). Thyroid hormones and their effects: a new perspective. Biol. Rev., 75, 519-631.

- Hurcombe, S.D. (2011). Hypothalamic-Pituitary gland axis function and disfunction in horses. Vet Clin Equine, 27, 1-17.

- Hyypp, S. (2005). Endocrinal responses in exercising horses. Livestock Production Science, 92, 113-121. 
- Irvine CHG, Evans MJ. (1975) Postnatal changes in total and free thyroxine and triiodothyronine in foal serum. $J$ Reprod Fertil Suppl, 23,709.

- Johnson, C.A. (2002). Thyroid Issues in Reproduction. Clin Tech Small Anim Pract, 17(3), 129-132.

- Johnson, P.J. (2002). The equine metabolic syndrome Peripheral Cushing's syndrome. Vet Clin Equine, 18, 271-293.

- Johnson, P.J.; Messer, N.T.; Slight, H.S.; Wiedmeyer, C.; Buff, P. \& Ganjam, V.K. (2004). Endocrinopathic Laminitis in the Horse. Vet Clin Equine, 3(1), 45-56.

- Kavazis, A.N.; Kivipelto, J. \& Ott, E.A. (2002). Supplementation of broodmares with copper, zinc, iron, manganese, cobalt, iodine, and selenium. Equine Vet $\mathrm{J}$, 22(10), 460-464.

- Malinowski, K.; Christensen, R.A.; Hafs, R.D. \& Scanes, CG. (1996). Age and breed differences in thyroid hormones, insulin-like growth factor (IGF)-I and IGF binding proteins in female horses. J Anim Sci, 74, 19361942.

- McLaughlin, B.G. \& Doige, C.E. (1982). A Study of Ossification of Carpal and Tarsal Bones in Normal and Hypothyroid Foals. Can Vet J, 23, 164-168.

- McLaughlin, B.G.; Doige, C.E. \& McLaughlin, P.S. (1986). Thyroid-hormone levels in foals with congenital musculoskeletal lesions. Can Vet J, 27, 264-7.

- Medica, P.; Cravana, C.; Fazio, E. \& Ferlazzo, A. (2011). 24-hour endocrine profiles of quarter horses under resting conditions. Equine Vet J, 31, 35-40.

- Messer N.T. \& Johnson, P.J. (2007). Evidence-Based Literature Pertaining to Thyroid Dysfunction and Cushing's Syndrome in the Horse. Vet Clin Equine, 23, 329-364.

- Messer, N.T.; Johnson, P.J.; Refsal, K.R. et al. (1995). Effect of food deprivation on baseline iodothyronine and cortisol concentrations in healthy, adult horses. Am J Vet Res, 56(1), 116-21.

- Messer, N.T.; Riddle, T.; Traub-Dargatz J.L.; Dargatz, D.A.; Refsal, K.J.; Thompson, D.L. (1998). Thyroid Hormone Levels in Thoroughbred Mares and Their Foals at Parturition. Proceedings of the Annual Convention of the AAEP. 44: 248-251.

- Murray, M.J. (1990). Hypothyroidism and respiratory insufficiency in a neonatal foal. J Am Vet Med Assoc,197(12), 1635-8.

- Nicassio, M.; Aiudi, G.; Silvestre, F.; Matarrese, R.; De Sandro, A.S. \& Lacalandra, G.M. (2008). Free thyroid hormone and cortisol levels in stallions during the breeding season. J Anim Sci, 107(3-4), 335-336.

- Roser, J.F. (2008). Regulation of testicular function in the stallion: An intricate network of endocrine, paracrine and autocrine systems. Anim Reprod Sci, 107, 179-196.

- Rudi's, P.; R'onai, Z. \& Bartha, T. (2005). Thyroid hormone metabolism in the brain of domestic animals. Domest Anim Endocrinol, 29, 88-96. 
- Sojka, J.E. (1993). Factors which affect serum T3 and T4 levels in the horse. Equine Pract, 15(10), 15-9.

- Symonds, M.E. (1995). Thyroid hormones and nutrient supplementation during pregnancy. Equine Vet Educ, 7(5), 246-8.

- Tahboub, R. \& Arafah, B.M. (2009). Sex steroids and the thyroid. Best Pract Res Clin Endocrinol Metab, 23(6), 769-780.

- Toribio, R.E. (2011). Endocrine dysregulation in critically ill foals and horses. Vet Clin North Am Equine Pract, 27(1), 35-47.

- Toribio, R.E. \& Duckett, W.M. (2004). Equine Internal Medicine. 2da ed. Estados Unidos de América: Saunders; 2004. p. 1342-1355.

- Walsh, D.N.; McGowan, C.M.; McGowan, T.; Lamb, S.V.; Schanbacher, B.J. \& Place, N.J. (2009). Correlation of Plasma Insulin Concentration with Laminitis Score in a Field Study of Equine Cushing's Disease and Equine Metabolic Syndrome. Equine Vet J, 29(2), 87-94.

- Warner, M.H. \& Beckett, G.J. (2010). Mechanisms behind the non-thyroidal illness syndrome: an update. J Endocrinol, 205, 1-13.

1. Laboratorio de Investigación en Bioquímica Clínica y Patología Molecular, Departamento de Ciencias Básicas de la Salud, Universidad de Caldas. jose.osorio_o@ucaldas.edu.co

2. Departamento de Salud Animal, Universidad de Caldas. 